\title{
Trajectories of Human Multi-Joint Arm Movements: Evidence of Joint Level Planning
}

\author{
J. Randall Flanagan and David J. Ostry \\ Department of Psychology. McGill University \\ Montreal, Quebec, Canada
}

\begin{abstract}
In this paper we show that staggered joint interpolation [7] with maximally smooth joint motion can account for both the hand paths and tangential velocity profiles observed in human multi-joint arm movements. Arm trajectories were recorded while subjects performed point-to-point movements in a vertical plane. The shape of the tangential velocity profile of the hand was found to be symmerical and bell-shaped. Although this finding is predicted by the maximum-smoothness theory [6], which assumes that hand motion is maximally smooth, the theory cannot account for the curved hand paths which were often observed. The present model assumes that joint motion, rather than the motion of the hand, is maximally smooth. The results of this work suggest that multi-joint amm movements may be planned at the joint level.
\end{abstract}

\section{Introduction}

The problem of trajectory planning is central to the study of human motor control as well as to the field of robotics. The challenge in robotics is to develop trajectory planning methods that are both flexible and efficient. In human motor control, the challenge is to discover how a working system, which is both flexible and efficient, works.

Recently, workers in human motor control have attempted to identify the coordinates in which human multi-joint arm movement trajectories are planned. Several investigators [1][6][9] have suggested that multi-joint arm 
movements are planned in cartesian endpoint coordinates whereas others [7][10] have argued that these movements are planned in joint angular coordinates. There are, of course, other possibilities. For example, multi-joint arm movement trajectories may be planned in terms of muscular coordinates. Moreover, movement trajectories may not be planned at all. Indeed, it has been suggested that the central nervous system may only specify the final configuration of the arm and that the trajectory towards the final position is determined by neuromuscular dynamics and by external constraints on the movement [3][4].

\section{Theory}

Early evidence for endpoint planning was provided by the work of Morasso and others [1][8][9] which investigated the trajectories of point-10-point human arm movements restricted to a horizontal plane. These movements were characterized by straight line hand paths in cartesian coordinates regardless of target position and movement rate. In addition, when subjects were instructed to simply move between targets, the shape of the tangential velocity profile of the hand was found to be bell-shaped and symmetrical. Some evidence for hand level planning has also been provided by the experiments of Soechting and Lacquaniti [10] in which the hand paths of pointing movements to targets at different heights were found to form straight lines. These findings may be interpreted as evidence for hand space planning.

This work led to the formulation of the maximum-smoothness theory or minimum-jerk theory by Flash and Hogan [6]. This theory posits that movements are organized to minimize mean squared jerk at the endpoint. In agreement with the empirical findings on horizontal arm movements, the maximum smoothness theory predicts symmetrical bell-shaped tangential velocity profiles at the movement endpoint and straight line endpoint paths. 
Whereas the maximum-smonthness theory car successfuliy describe the vajectories of human point-io-poin: horizonta! arm movements. the theor fail; to accouni fo: the crajectones of verical movements. Atkeson and Hollerbach [2] examined the kinematics of unrescained arm movements made between targets located in a vertical plane. ir parial agreement with the minimum-jerk theory, they found that the shape of the wrist tangential velocity profiles was bell-shaped and symmetrical independent of target position, movement rate, and even hand-held load. However, in contrast to the minimum-jerk theory, they reported both straight line and curved wrist paths; the curvature of the wrist path depended on the area of the workspace in which the movement occured. This finding is not predicted by the minimum-jerk theory.

In a later paper, Hollerbach and Atkeson [7] introduced the joint leve! planning strategy of staggered joint interpolation to account for both the curved and straight line hand paths observed in their experiments on vertical point-to-point arm movements as well as for the hand paths reported by others. Staggered joint interpolation is a generalized form of linear joint interpolation in which all joints have the same time profiles between the start and end of movement. However, in staggered joint interpolation, unlike linear joint interpolation, the joints are not required to start (or stop) moving at the same time.

Linearly interpolated motion is generally characterized by curved hand paths. However, staggering the joint start or end times can often result in near straight paths in endpoint space [7]. It is only when straight line hand paths would require a joint reversal that near straight hand paths cannot be achieved via staggered joint interpolation. Hollerbach and Atkeson [7] have demonstrated that curved hand paths are often observed under such conditions.

On the one hand, staggered joint interpolation appears to account well for both the straight and curved endpoint paths observed in point-to-point multi-joint movements. On the other hand, the tangential velocity profile of the movement endpoint has been found to be bell-shaped and symmerrical 
under a wide variety of experimental conditions, as predicted by the maximum-smoothness theon. In this paper we present a version of staggered joint interpolacion which can account for both the straight and curved hand paths and the bell-shaped symmetrical tangential velocity profiles that we have observed in our experiments. This version makes two assumptions: the joint angles follow minimum-jerk trajectories and the joints may be staggered at both the start and the end of the movement. To evaluate whether this version of staggered joint interpolation can account for the kinematics of point-to-point verical arm movements, we have simulated hand paths and tangential velocity profiles and compared the results to experimental records. In the simulations we have used empirically determined initial and final joint angles and joint movement start and end times. In addition, we have examined directly the experimental joint angular velocity profiles.

\section{Methods}

We have examised the trajectories of point-to-point arm movements made between various targets located in a vertical plane. Movements were performed at both a preferred rate and at a faster rate. Four subjects (three females and one male) were instructed to make single smooth movements between targets and were told to not make corrective adjustments near the end of the movement.

A pegboard was used to position the targets in a plane sagittal to the subject. Targets consisted of circular disks $(10 \mathrm{~cm}$. in diameter) placed on the ends of pegs. Subjects were required simply to position their finger beside the target disks at the star and end of the movement.

Movements were recorded in 3-D with the WATSMART infrared imaging system. IREDs (infrared emitting diodes) were placed at the proximal and distal end of the upper and lower arms and were used to 
reconstruct joint angles. Each IRED was sampled at $400 \mathrm{~Hz}$ and digitally filtered with a Butterworth low-pass filter with a cutoff frequency of $6 \mathrm{~Hz}$.

Spherical polar coordinates were used to define the position of the arm in 3-D (Figure 1). The position of the hand in space can be described by six parameters including four joint angles and the lengths of the upper and lower arms. The joint angles are upper arm (or shoulder) elevation $S$; upper arm yaw $\mathbf{Y}$, lower arm (or elbow) elevation $\mathbf{E}$; and upper arm roll $\mathbf{R}$. Other joint angular coordinates can, and have, been used to describe the position of the arm [11]. However, for the purposes of the present analysis, the angles chosen were deemed appropriate since they change monotonically during point-to-point vertical arm movements; a prerequisite for joint interpolation. Although we have defined the position of the arm in 3-D, it should be noted that these movement occurred largely in the sagittal plane. Consequently, the principal angles of interest are the shoulder and elbow angles.

SPHERICAL POLAR COORDINATES

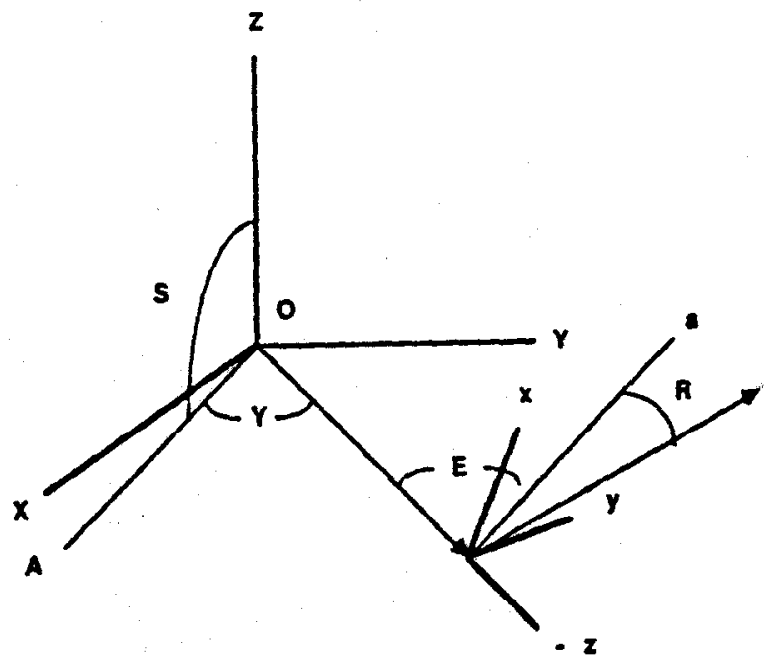

Figure 1: Amm position coordinates 


\section{Results}

\subsection{Experimental and Simulated Hand Paths}

Experimental hand paths for one subject are shown in Figure 2. With the exceprion of the vertical movement, hand paths are unaffected by movement direction between targets. Furhermore, band paths do not vary with movement speed. In the case of the vertical movement, the upward hand paths are more curved than the downward tand paths which are nearly straight. The hand paths observed in this study are similar to those reported by Atkeson and Hollerbach [2].

To assess bow well staggered joint interpolation coupled with minimum-jerk joint trajectories could account for the experimental movement trajectories, we carried out simulations. The initial and final values for the four joint angles shown in Figure 1 and the joint movement start and end times were scored from the empirical movement erajectories. Joint start and end times were determined on the basis of $10 \%$ of peak joint angular velocity. The motions of the four joint angles were assumed to follow minimum-jerk trajectories.

Simulated hand paths are presented in Figure 3. The initial and final joint angles and joint start and end times used in these simulations were taken from the experimental hand paths shown in Figure 2 (preferred speed). We assumed that the length of the lower arm was $25 \%$ greater than the length of the upper arm in these simulations. This number was based on direct measurements of our subjects.

The simulated hand paths capture many of the features of the experimental hand paths. However, there are some diserepancies. In particular, one can see that the inward movements for paths 1 and 2 are quite curved in contrast to the experimental data The reason for this will become clear in examining the joint velocity profiles (see Section 3.3). 


\section{0}

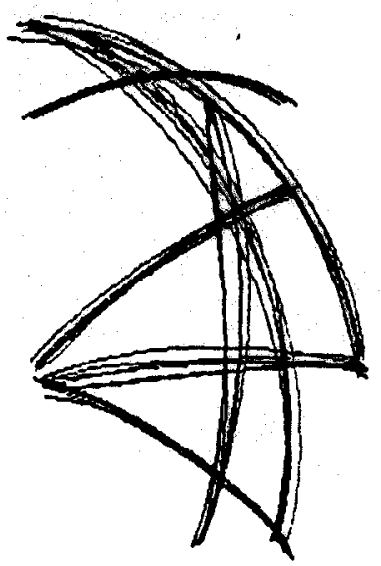

Prations

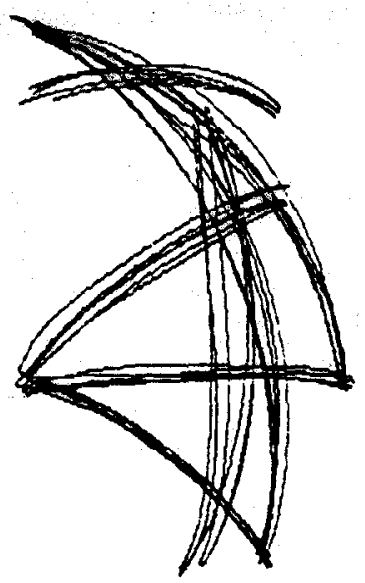

TAST

Figure 2: Experimental hand paths

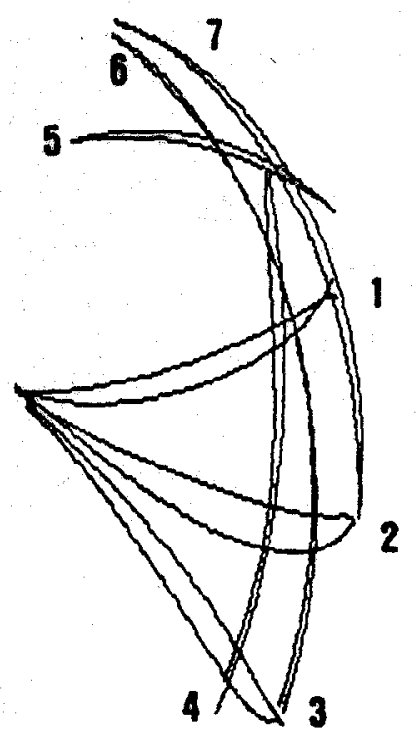

Figure 3: Simulated hand paths 


\subsection{Experimental and Simulated Hand Tangential Velocit! Profiles}

Experimental and simulated tangential velocity profiles of the hand are presented for paths 4 and 2 in Figure 4. For each path, velocity profiles are shown for both speed conditions and for both movement directions. Each set of velocity functions includes empirical (light traces) and simulated (heavy dashed trace) tangential velocity profiles together with a minimum-jerk velocity profile (heavy solid trace). Following Alkeson and Hollerbach [2], these functions have been normalized with respect to peak velocity and movement amplitude (i.e., area under the velocity curve) and shifted to minimize the area between the functions.

The experimental tangential velocity profiles shown for the vertical movements (path 4) are well approximated by both the minimum-jerk function and the simulated tangential velocity profiles based on staggered joint interpolation coupled with minimum-jerk joint trajectories and empirically determined joint movement amplitudes and durations. This holds across movement direction and rate. Similar tesults were found for movement paths $3,5,6$ and 7 .

For the horizontal movements (path 2) both the minimum-jerk function and the simulated tangential velocity profiles account well for the experimental velocity profiles in the case of the outwardly directed movements. However, the simulation fails to predict the experimental tangential velocity profiles in the case of the inwardly directed movements which are well described by the minimum-jerk function. The simulation also fails to account for the experimental velocity profiles observed for the inwardly directed movements of path 1. 

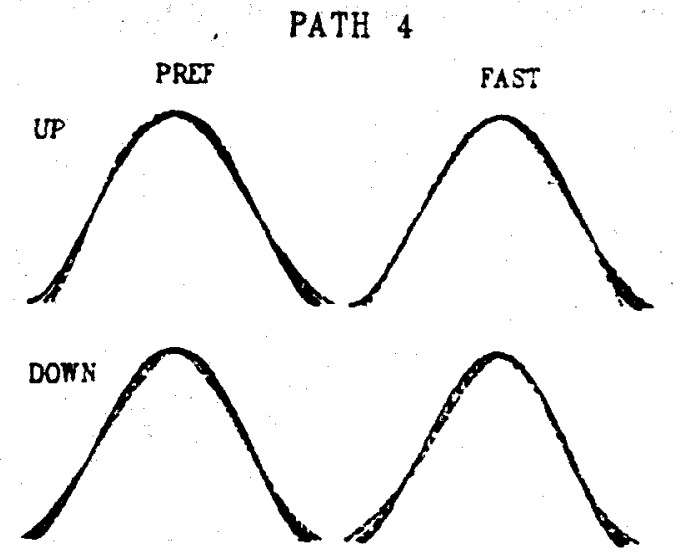

PATH 2

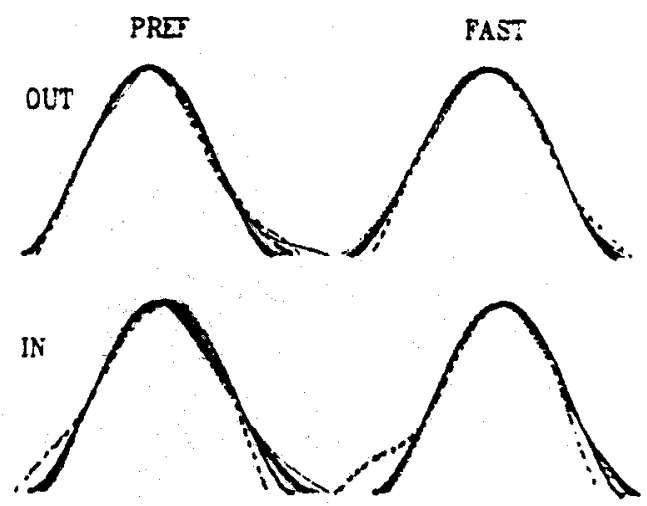

Figure 4: Tangential velocity profiles. Empirical (light), model (heavy dashed), and minimum-jerk (heavy solid) functions are shown.

In summary, the comparison of experimental and simulated hand paths and hand tangential velocity profiles demonstrates that, in general, the kinematics of point-10-point verical arm movements are well modelled by staggered joint interpolation with the assumption of minimum-jerk joint angle trajectories. However, there are some exceptions. To further investigate these movements, we assessed the form of the joint velocity profiles directly. 


\subsection{Experimental Joint Velocity Profiles}

Experimental shoulder and elbow velocity profiles (dotted traces) for horizontal (path 2) and vertical (path 4) moverments are presented in Figure 5. Minimum-jerk velocity functions (solid traces) have been included for reference. The empirical and minimum-jerk profiles have been normalized with respect to peak velocity and movement amplitude and shifted to minimize the area between the curves [2].

For the horizontal (path 2) outward movements, the elbow velocity profiles are positively skewed. In other words, the minimum-jerk profile (used to model joint velocity profiles in the simulations) is negatively skewed relative to the empirical records. In contrast, the shoulder velocity profiles are bell-shaped and symmetrical. Imporantly, the amplitude (and peak velocity) of the elbow movements is about twice as great as the shoulder movements (see Figure 6, upper panel) and consequently the form of the velocity profile of the elbow has a greater influence on the shape of the tangential velocity profile. Since the minimum-jerk function is negatively skewed relative to the elbow velocity profile, it is not suprising that the simulated tangential velocity profile is also negatively skewed relative to the experimental data.

A similar but opposite pattem is observed for the inward horizontal (path 2) movement. Once again, the amplitude of the elbow is double the amplitude of the shoulder (see Figure 6, middle panel). However, as shown in Figure 5, the elbow velocity profiles are now bell-shaped and symmetrical whereas the shoulder profiles are positively skewed. Since the elbow velocity profile is similar to the minimum-jerk function, the tangential velocity profile of the hand is well approximated by the simulation.

As noted above, the vertical (path 4) upwand movement features a curved hand path whereas the hand path of the vertical downward movement 
604
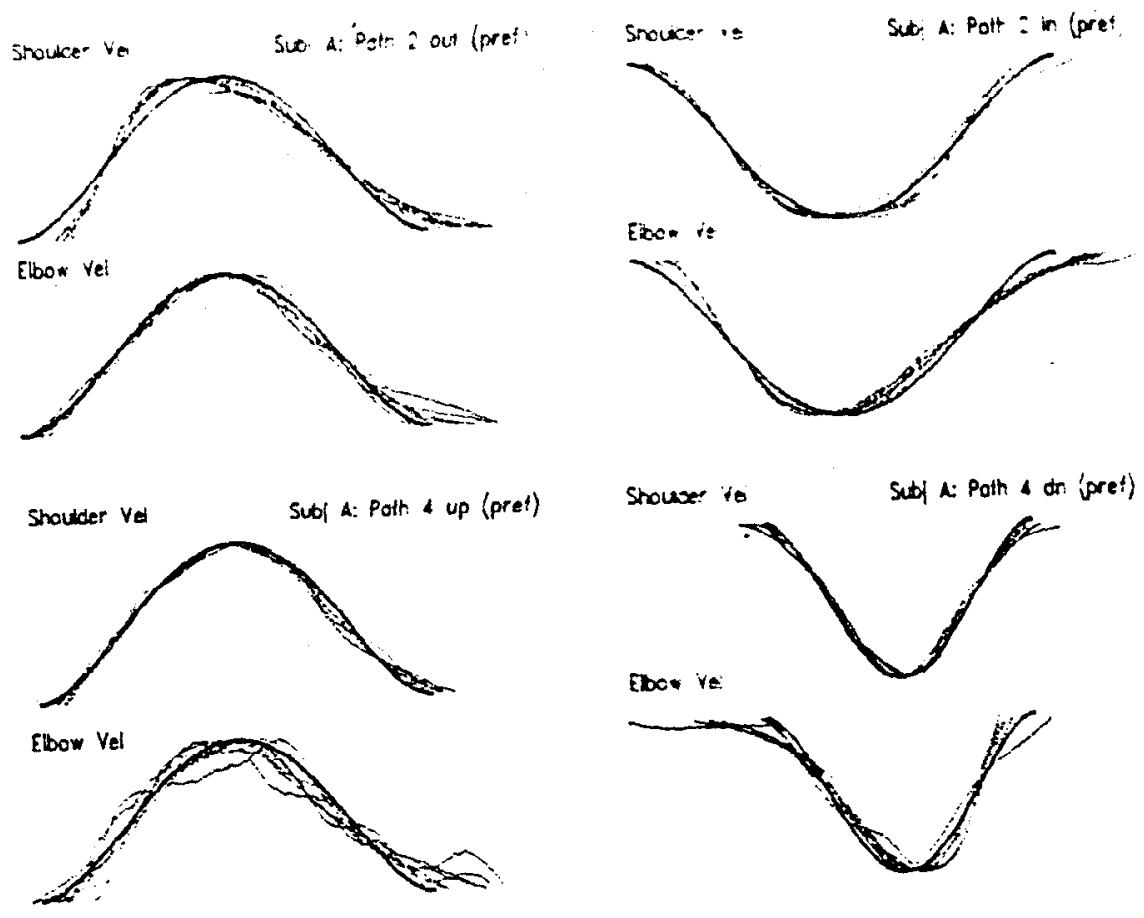

Figure 5: Joint angular velocity profiles. Empirical (dotted traces) and minimum-jerk (solid trace) functions are shown.

is closer to a straight line. The elbow and shoulder velocity profiles for these movements are presented in Figure 5. For both the upward and downward movements, the shoulder velocity profile is bell-shaped and symmetrical. The elbow velocity profile for the downward movement is also bell-shaped and symmetrical. In constrast, the elbow is essentially still 
Subj A: Poth 2 out (pref)

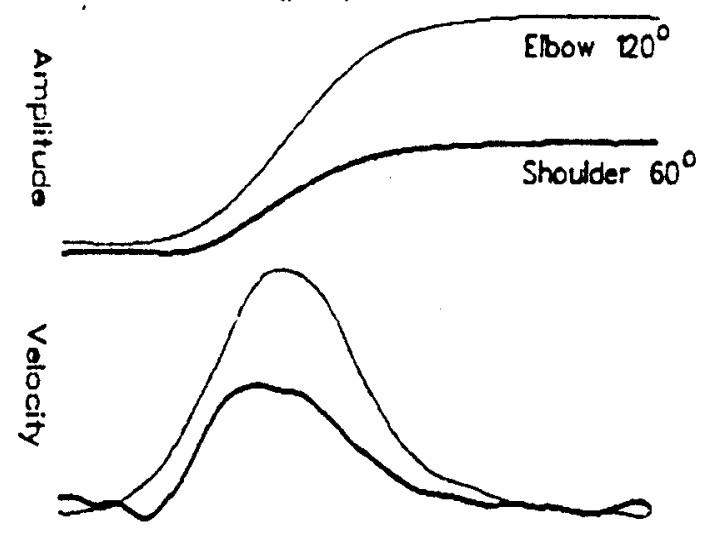

Subi A: Polh 2 in (pref)

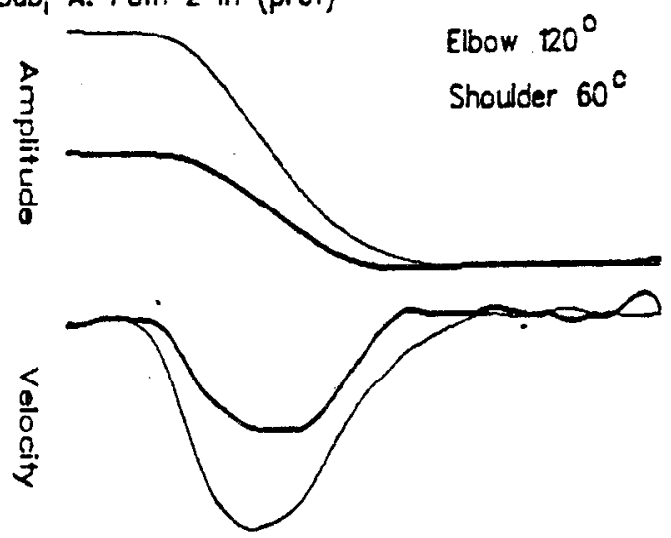

Subj A: Poth 4 dn (pref)

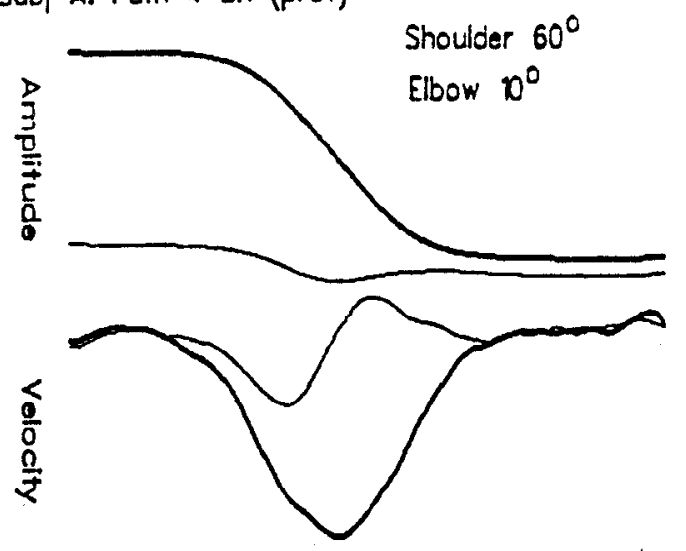

Figure 6: Elbow and shoulder amplitude and velocity 
during the upward movement and consequendy, the hand path is curved. A typical record of the vertical downward movement is shown in Figure 6 (lower panel). The small elbow flexion at the beginning of the movement accounts for the near straight hand path. This example shows how staggered joint motion can often produce near straight hand paths where curved paths would be seen given linear joint interpolation.

\subsection{Interaction of Joint and Hand Tangential Trajectories}

In linear joint interpolation, the path (but not the tangential velocity profile) of the endpoint is independent of the form of the joint velocity profiles. However, in staggered joint interpolation, both the path and the tangential velocity profile of the endpoint depend, in part, on the form of the angular velocity profiles at the joints. In order to investigate the relationship between the shape of the joint angular velocity profiles and the trajectory of the hand, we carried out simulations in which the arm was modelled as a two-joint planar manipulator with equal link lengths.

Figure 7 shows the joint and tangential velocity profiles for a linearly joint interpolated movement. The joints follow minimum-jerk trajectories and are shown in the same scale; the amplitude of the elbow is double that of the shoulder. The tangential velocity profile is shown in the middle panel. The lower panel shows a scored tangential velocity profile (movement start and end determined on the basis of $10 \%$ peak velocity) with a minimum-jerk profile overlaid. The tangential velociry profile was scored to simulate data analysis used in this and other studies. These profiles have been normalized with respect to peak velocity and amplitude and shifted to maximize the overlap between the curves (see [2]).

Although the joint velocity profiles shown in Figure 7 are bell-shaped and symmetrical, the resulting tangential velocity profile is skewed positively. However, when the tangential velocity profile is scored and normalized, it is 


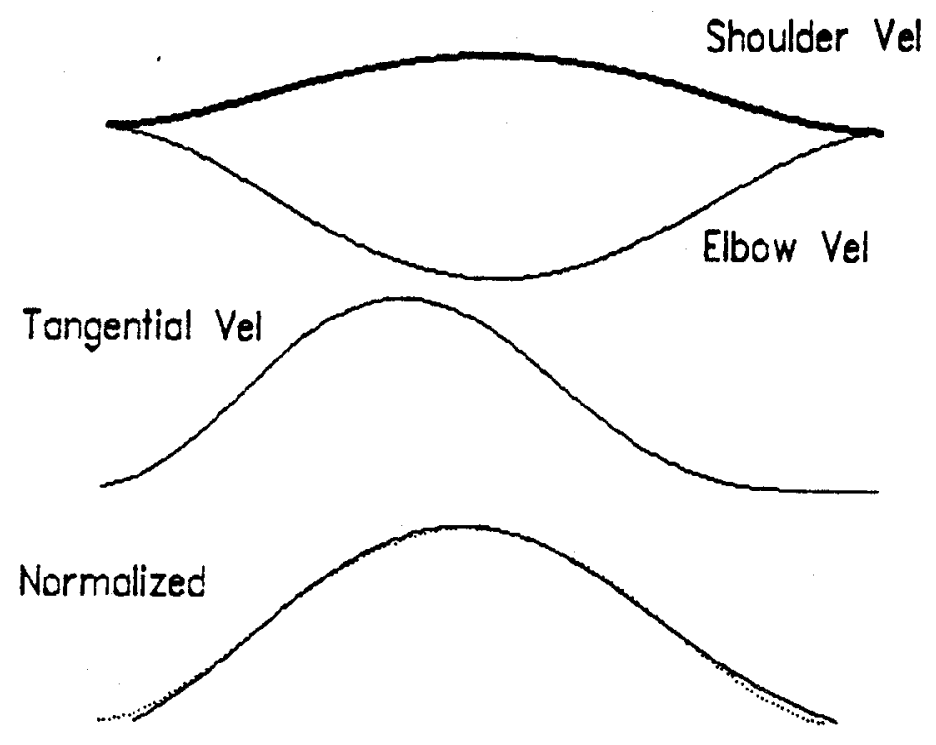

Figure 7: Simulated joint and tangential velocity profiles.

Maximally smooth joint motion with linear joint interpolation.

well approximated by a minimum-jerk function; this is a general characteristic of two-joint planar motion under linear joint interpolation.

Figure 8 illustrates the joint and tangential velocity profiles for joint interpolated movements in which elbow motion onset has been delayed by 0 , 10 and $20 \%$ of movement duration. The amplitude of shoulder flexion is equal to the amplitude of elbow extension in these movements. Consequently, under linear joint interpolation, the tangential velocity profile will have the same shape as the joint profiles. The movements illustrated in Figure 8 have minimum-jerk joint trajectories. The hand paths for these movements are shown in Figure 9 (upper panel). Whereas the joint onset staggering markedly effects the path of the hand, the effect on the tangential velocity profile of the hand is less clear. Only when the elbow onset is delayed by $20 \%$ of movement duration can a clear difference between the scored and normalized tangential velocity profile and the minimum-jerk 


\section{8}
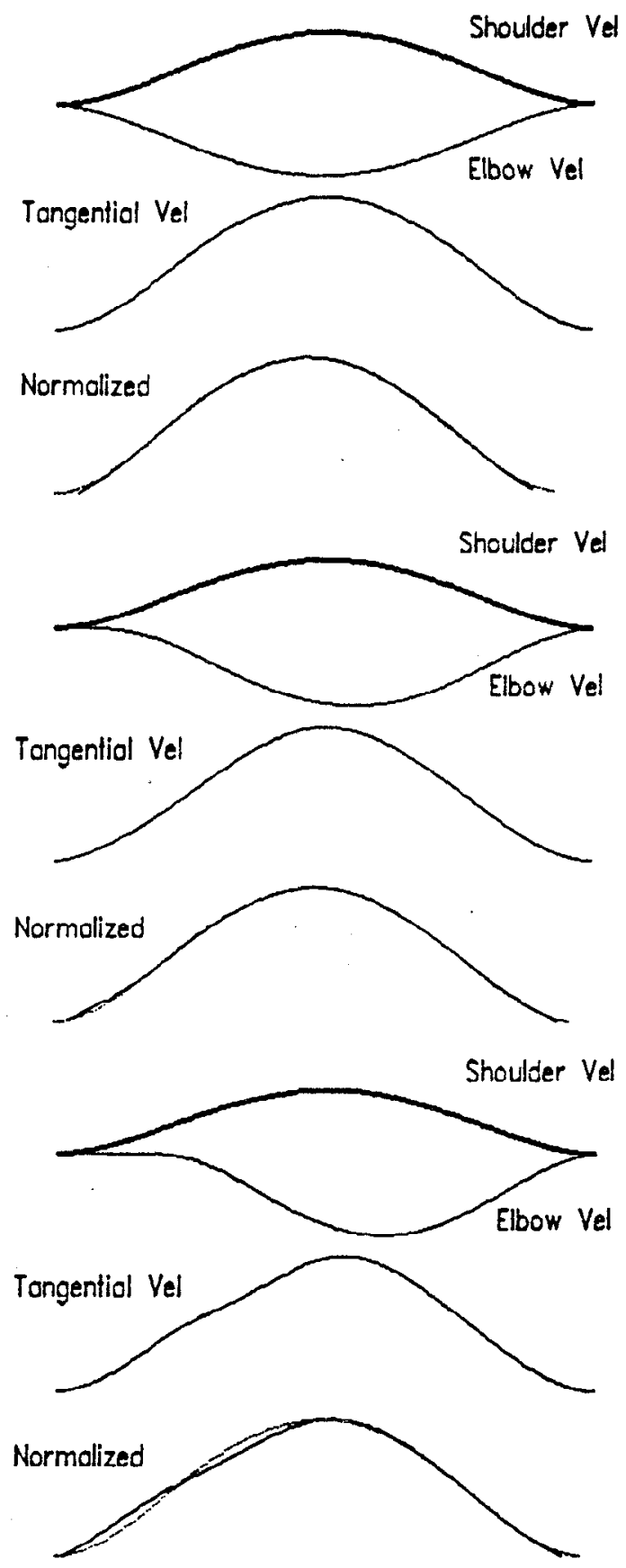

Figure 8: Simulated joint and tangential velocity profiles. Maximally smooth joint motion with $0 \%$ (upper 3 curves), $10 \%$ (middle 3 curves), and 20\% (lower 3 curves) initial elbow stagger. 


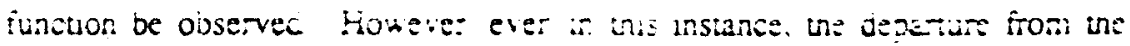
minimum-jeri pronite is no: grea:

Joint interpolater movements in which the join: velocts profiles are positively skewed are presented 1r. Figure 1C. A beia densig: juncion was used to simulate the skewed joint veiocity profiles. The figure shows movements in which eloow onse: has been delayed by 10 and $20 \%$ of

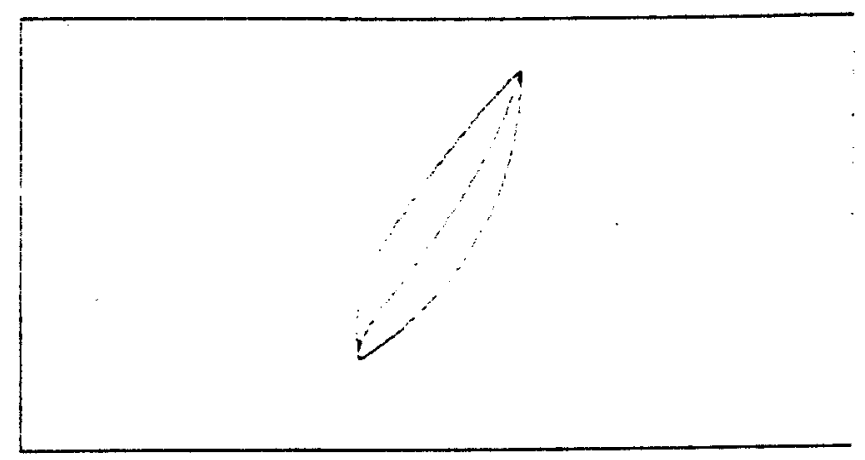

1/3, 1/20, Elbov stager (Left $\rightarrow$ Rignt)

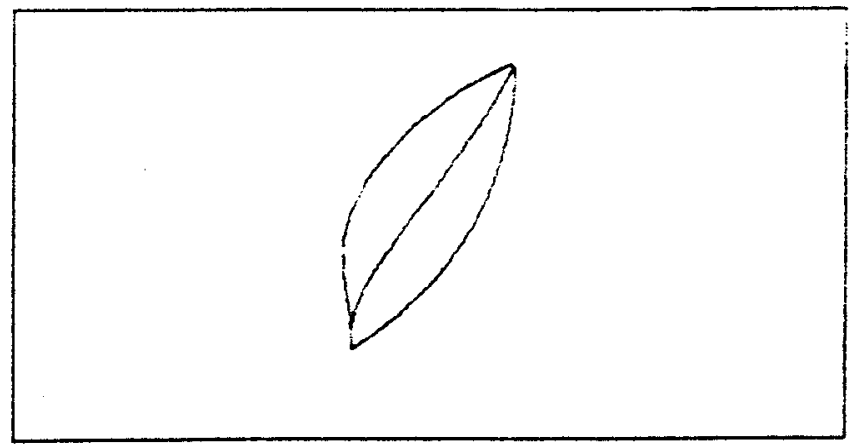

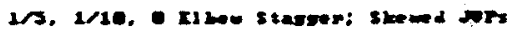

Figure 9: Simulated hand paths. Symmetrical (upper panel) and skewed (lower panel) joint velocity profiles with 20 , 10 and $0 \%$ (left to right) initial elbow stagger. 
movement duration. Once again, the path of the hand is strongly effected by the degree of elbow stagger (see Figure 9, lower panel). Comparison of the upper and lower panels of Figure 9 demonstrates the dependence of the hand path on the form of the joint velocity profiles. With $10 \%$ elbow stagger, the shape of the scored and normalized tangential velocity profile is reasonably well accounted for by the minimum-jerk function. However, when the
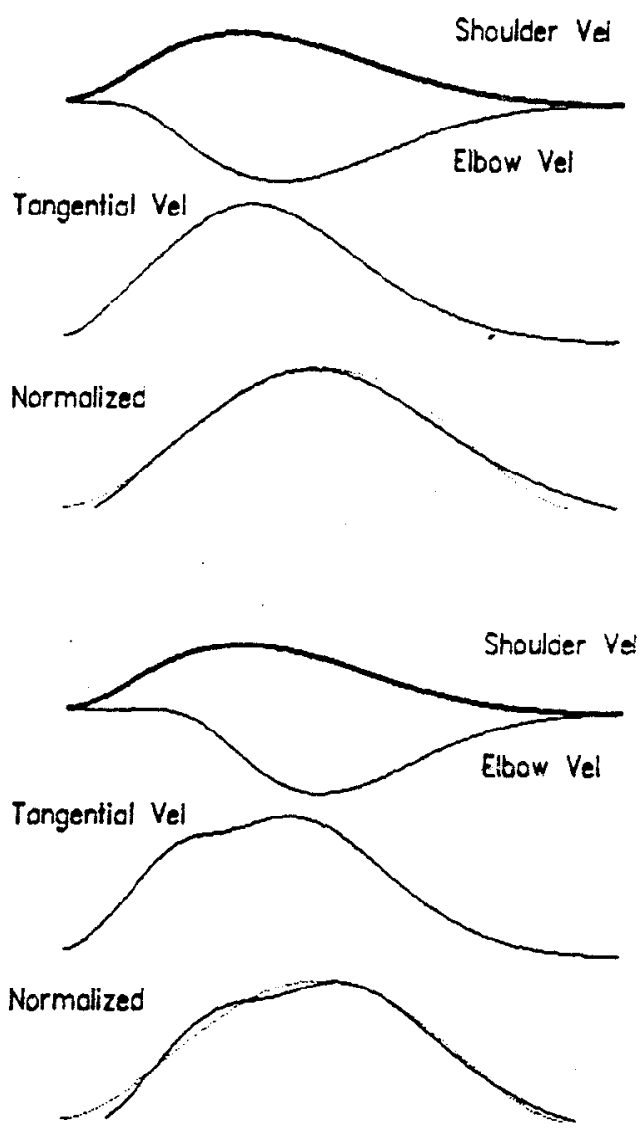

Figure 10: Simulated velocity profiles of skewed joint motion with $10 \%$ (upper panel) and $20 \%$ (lower panel) initial elbow stagger. 
skewed joint velocity profiles are combined with $20 \%$ stagger, the tangential velocity profile of the hand is clearly not bell-shaped or symmetrical.

\section{Conclusions}

We have examined the trajectories of point-to-point multi-joint arm movements made between targets in a vertical plane. In agreement with previous work [2] the shape of the tangential velocity profile of the hand was found to be bell-shaped and symmetrical and the curvature of hand path was found to depend on target location in the workspace. We have shown that, in general, both the form of the hand path and the shape of the tangential velocity profile can be modelled with a version of staggered joint interpolation in which the joints are assumed to follow maximally smooth trajectories.

To test this model, we carried out simulations in which the initial and final joint angles and the joint start and end times were taken from experimental records and the joints were made to follow minimum-jerk trajectories. In most cases, the simulated hand paths and tangential velocity profiles agreed well with the empirical data. However, the model failed to account for the hand paths and tangential velocity profiles observed for inwardly directed horizontal movements. On examination of the experimental joint angular velocity profiles of these movements, it was found that the elbow velocity profile was positively skewed Consequently, the assumption of bell-shaped and symmetrical (i.e., minimum-jerk) joint trajectories made in the model was inappropriate for these movements.

The finding that minimum-jerk joint trajectories generally correspond to tangential velocity profiles which are similar in form seems suprising since the mapping between joint velocity and hand velocity is non-linear. However, simulations showed that, under linear joint interpolation with 
minimum-jerk joint velocity profiles, tangential velocity profiles remain approximately bell-shaped and symmetrical when the tangential velocity profiles are scored using standard techniques (see Section 3.4). Even when joint staggering is introduced, the tangential velocity profiles are often reasonably bell-shaped and symmetrical. However, if the joint velocity profiles are skewed then the tangential velocity profile will tend not to be bell-shaped and symmetrical.

In summary, the kinematics of the vertical arm movements examined in this study can be well accounted for by a model combining staggered joint interpolation and minimum-jerk joint trajectories. This finding suggests that human arm movements are planned, at least in par, in joint coordinates.

\section{References}

[1] Abend, W., Bizzi, E. and Morasso, P., 1982. Human arm trajeciory formation. Brain, 105, 331-348.

[2] Atkeson, C.G. and Hollerbach, J.M., 1985. Kinematic features of unrestrained vertical arm movements. J. Neurosci., 9, 2318-2330.

[3] Berkinblit, M.B., Feldman, A.G. and Fukson, O.I., 1986. Adaptability of innate motor patterns and motor control mechanisms. Behav. Brain Sci., 9, 585-638.

[4] Bizzi, E., Chapple, W. and Hogan, N., 1982. Mechanical properties of muscles: implications for motor control. Trends Neurosci., 5, 395-398.

[5] Flanagan, J.R. and Ostry, D.J., 1988. Kinematics of two and three link sagittal arm and arm with pointer movements. Soc. Neurosci. Abstr., 14, 951. 
[6] Flash, T. and Hogan, N., 1985. The coordination of arm movements: An experimentally confirmed mathematical model. J. Neurosci., 7, 1688-1703.

[7] Hollerbach, J.M. and Atkeson, C.G., 1987. Deducing planning variables from experimental arm trajectories: Pitfalls and possibilities. Biol. Cybern., 56, 279-292.

[8] Hollerbach, J.M. and Flash, T., 1982. Dynamic interactions between limb segments between during planar arm movement. Biol. Cybern., 44, 67-77.

[9] Morasso, P., 1981. Spatial control of amm movements. Exp. Brain Res.. 42, 223-227.

[10] Soechting, J.F. and Lacquaniti, F., 1981. Invariant characteristics of a pointing movement in man. J. Neurosci., 1, 710-720.

[11] Soechting, J.F. and Ross, B., 1984. Psychophysical determination of coordinate representation of human arm orientation. Neurosci., 13, 595-604. 